\title{
Targeting ticagrelor: a novel therapy for emergency reversal
}

\author{
Brandon Cave $^{1}$, Aranyak Rawal ${ }^{2}$, Devarshi Ardeshna ${ }^{3}$, Uzoma N. Ibebuogu ${ }^{4}$, Chittoor B. Sai-Sudhakar ${ }^{5}$, \\ Rami N. Khouzam ${ }^{4}$
}

${ }^{1}$ Department of Pharmacy, Methodist University Hospital, Memphis, TN, USA; ${ }^{2}$ Department of Medicine-Pediatrics, ${ }^{3}$ College of Medicine, University of Tennessee Health Science Center, Memphis, TN, USA; ${ }^{4}$ Division of Cardiovascular Diseases, Department of Internal Medicine, University of Tennessee Health Science Center, Memphis, TN, USA; ${ }^{5}$ Department of Cardiac Surgery, University of Tennessee Health Science Center, Memphis, TN, USA

Contributions: (I) Conception and design: B Cave; (II) Administrative support: UN Ibebuogu; (III) Provision of study materials or patients: All authors; (IV) Collection and assembly of data: All authors; (V) Data analysis and interpretation: All authors; (VI) Manuscript writing: All authors; (VII) Final approval of manuscript: All authors.

Correspondence to: Brandon Cave. Pharm D, BCCP, ASH-CHC. Department of Pharmacy, Methodist University Hospital, 1265 Union Ave., Memphis, TN 38104, USA. Email: Brandon.cave@mlh.org.

\begin{abstract}
Newer P2Y12 inhibitors are prescribed in place of clopidogrel for patients with acute coronary syndrome (ACS) and are associated with significant bleeding risks. Currently, limited options exist for the management of life-threatening bleeding or acute reversal for patients on P2Y12 inhibitor therapy, specifically ticagrelor. Various interventions, including platelet transfusion and desmopressin, have been studied for ticagrelor reversal demonstrating limited success. PB2452 is a novel monoclonal antibody which binds to both ticagrelor and its active metabolite resulting in a rapid return of platelet aggregation. PB2452 has been studied in animal models and, most recently, in a Phase I trial in healthy volunteers. In animal models, PB2452 displayed rapid reversal of ticagrelor and its metabolites and return to near normal levels of platelet aggregation within $60 \mathrm{~min}$. In healthy human volunteers, cohorts that received higher dose bolus and infusions of PB2452 over 12-16 h resulted in maximal and sustained reversal of ticagrelor inhibition of platelet aggregation. While it is currently not US Food and Drug Administration approved, future Phase 2 and 3 studies are currently underway that may lead to new directions for patients on ticagrelor therapy who require urgent reversal.
\end{abstract}

Keywords: Ticagrelor; PB2452; antiplatelet reversal emergency reversal

Submitted Jun 30, 2019. Accepted for publication Jul 29, 2019.

doi: 10.21037/atm.2019.08.08

View this article at: http://dx.doi.org/10.21037/atm.2019.08.08

\section{Introduction}

Potent P2Y12 inhibitors, prasugrel and ticagrelor, are routinely being prescribed in place of clopidogrel for patients with acute coronary syndrome (ACS) due to established superior efficacy in ACS with or without percutaneous coronary intervention (1-3). More potent platelet inhibition translates into an increased risk of bleeding. However, a net clinical benefit favors the use of these agents and has been incorporated in numerous guidelines (4-6).

In the Platelet Inhibition and Patient Outcomes (PLATO) trial, ticagrelor increased PLATO-defined non-
CABG-related major bleeds (4.5\% vs. 3.8\%; HR 1.19, $95 \%$ CI, 1.02-1.38, $\mathrm{P}=0.03$ ) compared to clopidogrel as part of a dual antiplatelet regimen (2). There was no difference in life-threatening or fatal bleeding between ticagrelor or clopidogrel-treated patients. However, ticagrelor-treated patients who experienced intracranial bleeding were less likely to survive the event compared to clopidogrel $(0.1 \%$ vs. $0.01 \% ; \mathrm{P}=0.02$ ). Although these events were rare, it may suggest that these events are more devastating and fatal with ticagrelor.

Ticagrelor is unique in that it reversibly binds to the P2Y12 receptor and requires shorter interruption compared 
to prasugrel ( 3 vs. 7 days) prior to surgical intervention (7). Frequently, patients receive dual antiplatelet therapy (DAPT) upon presentation with suspicion for nonST segment elevation-ACS prior to knowledge of coronary anatomy, and if determined to be candidates for coronary artery bypass surgery, intervention is often delayed. Management strategies are complex in patients with hemodynamic instability, ongoing ischemia, critical coronary anatomy or high risk for recurrent ischemic events, as these are indications for emergent or urgent surgery without the luxury of waiting for full platelet recovery (4). Challenges also exist in patients with recent placement of a drug-eluting stent on DAPT with an urgent need for surgical intervention or invasive procedure, in which the risk of stent thrombosis is extremely high if administration of DAPT is temporarily discontinued or interrupted and bridging with intravenous cangrelor or GPIIb/IIIa inhibitors present a significant financial burden. At present there are limited options to address either urgent reversal for surgical procedures or life-threatening bleeding associated with P212 inhibitors, namely ticagrelor.

\section{Current strategies for ticagrelor reversal}

Current strategies to reduce the effects of antiplatelet drugs are limited. It has been suggested that platelet transfusion may be helpful, however, the mechanism of action of ticagrelor poses challenges with this strategy (8). The reversibility of ticagrelor's inhibition of P2Y12 allows unbound ticagrelor and its active metabolite to inhibit fresh platelets (8). Reports highlight platelet transfusions, even at high-doses, have been unsuccessful in patients with intracranial hemorrhage previously treated with ticagrelor $(9,10)$. In vitro studies have suggested that while pooled platelets are ineffective, platelet-rich plasma (PRP) may be more effective (8). The success of PRP is possibly attributed to the addition of plasma proteins, as demonstrated by an increase in platelet function with administration of human serum alone. Based on these results, it may be feasible to achieve similar effects with conventional doses of 20-40 g of human albumin to increase protein binding of ticagrelor.

Off-label use of desmopressin (DDAVP), a synthetic analogue of antidiuretic hormone, has been used in reversing ticagrelor-associated bleeding (11). DDAVP increases plasma factor VIII and von Willebrand factor concentration which promotes hemostasis $(12,13)$. DDAVP may often be used as first-line treatment for patients with bleeding disorders and it has demonstrated efficacy in reversing bleeding related to heparin, aspirin and clopidogrel (14-16). A randomized crossover study of healthy volunteers on ticagrelor showed that DDAVP administration increased the primary hemostatic activity, and lowered bleeding time from 10.5 to $7.5 \mathrm{~min}$, however, this difference was not statistically significant (11). DDAVP did not reverse the ticagrelor-associated inhibition of platelet aggregation. The results did not translate into clinical relevance. Without a definitive method of reversal, a specific antidote for ticagrelor may prove valuable as an agent for patients who require emergency procedures or have life-threatening bleeding. An antidote, human Fab MEDI2452, is currently under development and has demonstrable ticagrelor reversal in human in-vitro studies and mice in-vivo studies (17).

\section{Pharmacology}

PB2452 (formerly MEDI2452), a recombinant human monoclonal antibody antigen-binding fragment (Fab), has a dual mechanism of action in that it binds both to ticagrelor and its major active metabolite (AR-C124910XX) allowing restoration of platelet aggregation (17). It binds with strong affinity, approximately 100 -fold greater than ticagrelor to its P2Y12 receptor. Adenosine triphosphate was used as the blueprint to develop PB2452. Despite structural similarity to adenosine, PB2452 remains highly targetspecific and does not display the same binding affinity to adenosine or like compounds (17). PB2452 is only able to bind free ticagrelor and continues to bind ticagrelor as it dissociates from the P2Y12 receptor or plasma proteins. For this reason, sufficient time is necessary to achieve full reversal. Ticagrelor is primarily eliminated through the gastrointestinal tract. However, the complex of ticagrelor and PB2452 is eliminated primarily by the renal route. PB2452 represents the first in its class for reversing the effects of potent $\mathrm{P} 2 \mathrm{Y} 12$ inhibitors.

\section{Evidence of ticagrelor reversal}

\section{Animal models}

PB2452 has been evaluated in several animal models, including mice and pigs. To simulate reversal of major bleeding, Swedish landrace pigs were pretreated with aspirin and ticagrelor followed by partial liver lobectomy (18). Pigs were treated with $250 \mathrm{mg}$ of aspirin for two days prior to surgery and $10 \mathrm{mg} / \mathrm{kg}$ intravenously on the day of surgery. Ticagrelor was administered by a $60-$ min infusion of 


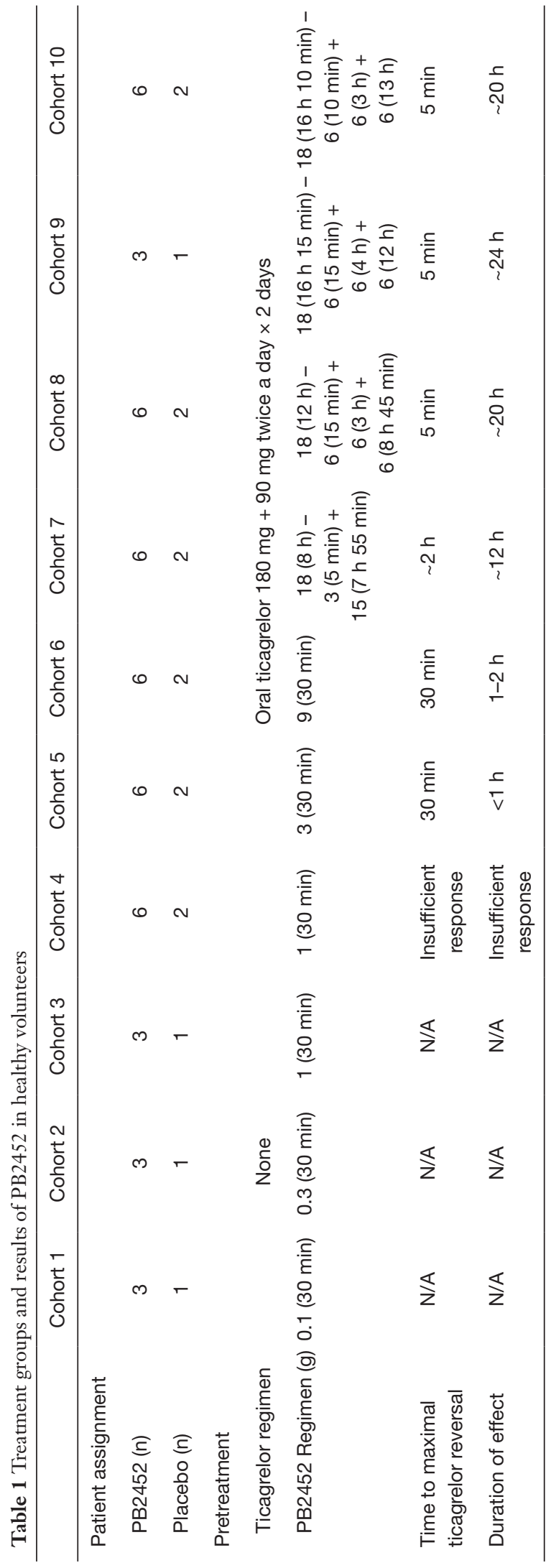

$3 \mathrm{mg} / \mathrm{kg}$ and terminated just prior to liver injury. Following surgical removal of partial lobe, a $600 \mathrm{mg} / \mathrm{kg}$ dose of PB2452 was administered over $5 \mathrm{~min}$. PB2452 eliminated free ticagrelor and AR-C124910XX within 5 min and returned platelet aggregation to near normal in approximately $60 \mathrm{~min}$, despite the experimental dosing regimen producing supratherapeutic concentrations of twice that of standard dosing in humans. Treatment with PB2452 showed a trend toward survival (70\% vs. 44\%) and survival time (240 vs. $169 \mathrm{~min}$ ), however, neither of these achieved statistical significance due to the small population. Rate of mean arterial pressure decrease was significantly diminished in pigs that received $\mathrm{PB} 2452,0.08$ vs. $0.141 \mathrm{mmHg}$ per min in pigs without reversal. These trends suggest the feasibility of reversal and require further evaluation in human subjects.

\section{Healthy volunteers}

In a phase 1, randomized, double-blind, placebo-controlled trial, PB2452 was evaluated for safety and efficacy in healthy volunteers (19). The primary outcome evaluated was ticagrelor reversal assessed by platelet aggregation through light transmission aggregometry at various times prior to and after PB2452 administration. Additional secondary assessments of ticagrelor reversal were performed with a point-of-care P2Y12 assay, and a vasodilator-stimulated phosphoprotein (VASP) phosphorylation immunoassay. The primary safety endpoint evaluated was the frequency and severity of adverse events.

Healthy volunteers between the ages of 18 and 50 years, weight between 50 and $120 \mathrm{~kg}$, and body-mass index between 18 and 35 were eligible for participation. Exclusion criteria included any contraindication to ticagrelor, predisposition to bleeding, or an estimated glomerular filtration rate $<60 \mathrm{~mL} / \mathrm{min} / 1.73 \mathrm{~m}^{2}$. Randomization occurred in a 3:1 fashion of PB2452 and placebo into 10 cohorts (Table 1). A total of 64 volunteers were included of which 48 received PB2452 and 16 received placebo. Cohorts 1-3 were not given pretreatment with ticagrelor and given low doses of PB2452 over $30 \mathrm{~min}$ $(0.1-1 \mathrm{~g})$. Cohorts 4-10 were pretreated with ticagrelor and given larger, increasing doses of PB2452. Cohorts 4-6 were given loading doses of 1,3 or $9 \mathrm{~g}$ over $30 \mathrm{~min}$. Cohorts 7-10 received a total of $18 \mathrm{~g}$ of $\mathrm{PB} 2452$, in which loading doses and duration of infusions varied.

The efficacy analysis was only conducted in those patients who received ticagrelor pretreatment (Cohorts $4-10)$. In volunteers that received placebo, platelet function 
returned to near baseline in 24-48 h. Cohort 4 had minimal response with $1 \mathrm{~g}$ of PB2452. Cohorts 5 and 6 achieved maximum ticagrelor reversal after the termination of the infusion at $30 \mathrm{~min}$, however, this effect was sustained for only $1-2 \mathrm{~h}$. Cohort 7 had sustained ticagrelor inhibition for $12 \mathrm{~h}$ with maintenance infusion, however, due to the lower loading dose, adequate onset was delayed for $2 \mathrm{~h}$. Cohorts 8-10 were given $18 \mathrm{~g}$ administered over $12 \mathrm{~h}$ (Cohort 8) or $16 \mathrm{~h}$ (Cohorts 9 and 10) and with larger $6 \mathrm{~g}$ boluses at initiation. These regimens displayed maximal ticagrelor reversal within $5 \mathrm{~min}$ and sustained effect over 16-24 h by light transmission aggregometry, P2Y12 platelet-reactivity, and VASP assays. Additional tests for a rebound prothrombotic effect with PB2452 were negative.

Adverse events occurred more frequently in PB2452treated patients ( $35 \%$ vs. 12\%), with bruising at the injection site being the most common. There were no deaths, thrombotic events, infusion-related reactions, or doselimiting toxic effects. PB2452 is a monoclonal antibody, and immunogenicity is a potential concern. Detectable antidrug antibodies were found in $44 \%$ of volunteers. However, only $12 \%$ became positive after receiving PB2452. Titers were low and did not show evidence of affecting safety or efficacy of PB2452. These results evaluating safety and efficacy are promising but will need confirmation in a larger trial of patients with coronary disease requiring cessation of antiplatelet therapies for surgery or with those active major bleeding.

\section{Future development}

At this time, PB2452 is not US Food and Drug Administration (FDA) approved; however, in 2019, the FDA granted Breakthrough Therapy designation for PB2452 based on Phase 1 and preclinical studies. Breakthrough Designation will allow for expedited development, review, communication and guidance from the FDA and future potential of Accelerated Approval and Priority Review (20). Recruitment to begin Phase 2a study in healthy older and elderly patients (aged 50-80 years) to evaluate safety, tolerability and pharmacokinetics and pharmacodynamics is currently underway (NCT03928353) (21).

\section{Conclusions}

Despite the routine use of potent P2Y12 inhibitors and investment into reversal therapies for novel anticoagulants, there is a lack of therapeutic options to effectively reverse antiplatelet therapy. This unmet need may continue to grow as combinations of anticoagulation and antiplatelet therapies increase bleeding risk when indications overlap. Although rare, the consequences of a major bleed from ticagrelor can be severe, and platelet infusions are ineffective. The addition of a reversal agent for ticagrelor will increase the safety of patients requiring reversal for acute uncontrolled or life-threatening major bleeding events. PB2452 represents an advancement in this area and is the first reversal agent of its kind in the case of urgent surgical procedures or uncontrolled major or lifethreatening bleeding.

\section{Acknowledgments}

None.

\section{Footnote}

Conflicts of Interest: Dr. Cave discloses that he serves on the speakers bureau for Portola Pharmaceuticals. The other authors have no conflicts of interest to declare.

Ethical Statement: The authors are accountable for all aspects of the work in ensuring that questions related to the accuracy or integrity of any part of the work are appropriately investigated and resolved.

\section{References}

1. Wiviott SD, Braunwald E, McCabe CH, et al. Prasugrel versus clopidogrel in patients with acute coronary syndromes. N Engl J Med 2007;357:2001-15.

2. Wallentin L, Becker RC, Budaj A, et al. Ticagrelor versus clopidogrel in patients with acute coronary syndromes. $\mathrm{N}$ Engl J Med 2009;361:1045-57.

3. Mahaffey KW, Wojdyla DM, Carroll K, et al. Ticagrelor compared with clopidogrel by geographic region in the Platelet Inhibition and Patient Outcomes (PLATO) trial. Circulation 2011;124:544-54.

4. Roffi M, Patrono C, Collet JP, et al. 2015 ESC Guidelines for the management of acute coronary syndromes in patients presenting without persistent ST-segment elevation: Task Force for the Management of Acute Coronary Syndromes in Patients Presenting without Persistent ST-Segment Elevation of the European Society of Cardiology (ESC). Eur Heart J 2016;37:267-315.

5. Kulik A, Ruel M, Jneid H, et al. Secondary prevention 
after coronary artery bypass graft surgery: a scientific statement from the American Heart Association.

Circulation 2015;131:927-64.

6. Levine GN, Bates ER, Bittl JA, et al. 2016 ACC/ AHA Guideline Focused Update on Duration of Dual Antiplatelet Therapy in Patients With Coronary Artery Disease: A Report of the American College of Cardiology/ American Heart Association Task Force on Clinical Practice Guidelines. J Am Coll Cardiol 2016;68:1082-115.

7. Valgimigli M, Bueno H, Byrne RA, et al. 2017 ESC focused update on dual antiplatelet therapy in coronary artery disease developed in collaboration with EACTS: The Task Force for dual antiplatelet therapy in coronary artery disease of the European Society of Cardiology (ESC) and of the European Association for Cardio-Thoracic Surgery (EACTS). Eur Heart J 2018;39:213-60.

8. Schoener L, Jellinghaus S, Richter B, et al. Reversal of the platelet inhibitory effect of the P2Y inhibitors clopidogrel, prasugrel, and ticagrelor in vitro: a new approach to an old issue. Clin Res Cardiol 2017;106:868-74.

9. Godier A, Taylor G, Gaussem P. Inefficacy of platelet transfusion to reverse ticagrelor. N Engl J Med 2015;372:196-7.

10. Willeman T, Marlu R, Böhle H, et al. Lethal cerebral hemorrhage after ticagrelor intoxication: a specific antidote is urgently needed. Clin Toxicol (Phila) 2018;56:1200-3.

11. Teng R, Mitchell PD, Butler K. The effect of desmopressin on bleeding time and platelet aggregation in healthy volunteers administered ticagrelor. J Clin Pharm Ther 2014;39:186-91.

12. Hashemi S, Palmer DS, Aye MT, et al. Platelet-activating factor secreted by DDAVP-treated monocytes mediates von Willebrand factor release from endothelial cells. J Cell
Physiol 1993;154:496-505.

13. Lange M, Van Aken H, Westphal M. Prevention and treatment of major blood loss. N Engl J Med 2007;357:1260; author reply 1261.

14. Teng R. Ticagrelor: Pharmacokinetic, Pharmacodynamic and Pharmacogenetic Profile: An Update. Clin Pharmacokinet 2015;54:1125-38.

15. Leithäuser B, Zielske D, Seyfert UT, et al. Effects of desmopressin on platelet membrane glycoproteins and platelet aggregation in volunteers on clopidogrel. Clin Hemorheol Microcirc 2008;39:293-302.

16. Mannucci PM. Desmopressin: a nontransfusional form of treatment for congenital and acquired bleeding disorders. Blood 1988;72:1449-55.

17. Buchanan A, Newton P, Pehrsson S, et al. Structural and functional characterization of a specific antidote for ticagrelor. Blood 2015;125:3484-90.

18. Pehrsson S, Johansson KJ, Janefeldt A, et al. Hemostatic effects of the ticagrelor antidote MEDI2452 in pigs treated with ticagrelor on a background of aspirin. J Thromb Haemost 2017;15:1213-22.

19. Ibrahim K, Christoph M, Quick S. Antibody-Based Ticagrelor Reversal Agent in Healthy Volunteers. N Engl J Med 2019;381:585-6.

20. PhaseBio Receives FDA Breakthrough Therapy Designation for PB2452 for the Reversal of the Antiplatelet Activity of Ticagrelor. Available online: https://investors.phasebio.com/news-releases/newsrelease-details/phasebio-receives-fda-breakthroughtherapy-designation-pb2452

21. PhaseBio Pharmaceuticals. Study to Evaluate the Safety, Tolerability, PK and PD of PB2542 in Older and Elderly Subjects. Accessed June 06, 2019. Available online: https:// www.clinicaltrials.gov/ct2/show/NCT03928353
Cite this article as: Cave B, Rawal A, Ardeshna D, Ibebuogu UN, Sai-Sudhakar CB, Khouzam RN. Targeting ticagrelor: a novel therapy for emergency reversal. Ann Transl Med 2019;7(17):410. doi: 10.21037/atm.2019.08.08 